and this has risen over the past year to $95 \%$. The ACHS provides data for 63 hospitals (both public and private) Australia-wide and the current overall compliance figure for this clinical indicator is $84 \%$.

We note that the service reported by Welthagen et al provided assessment of $22 \%$ of patients after a median time of 22 days. Although their service is a step in the right direction, we believe it falls far short of an ideal assessment of the physical status of those with mental disorders.

AUSTRALIAN COUNCIL OF HEALTHCARE STANDARDS (2003) Clinical Indicator Users' Manual 2004: Mental Health Indicators Version 4. Sydney: ACHS.

GOLDNEY, R., FISHER, L., WALMSLEY, S. (1998) Quality improvement by use of Clinical Indicators in a psychiatric hospital. Australasian Psychiatry, 6 191-193.

*Laura Fisher Research Officer, The Adelaide Clinic, Ramsay Health Care Mental Health Services South Australia, 33 ParkTerrace, Gilberton, South Australia, 5081, Robert Goldney Professor, The University of Adelaide, Psychiatry Department, The Adelaide Clinic, Gilberton, South Australia

\section{Bullying in the guise of career advice}

Hoosen \& Callaghan (Psychiatric Bulletin, June 2004, 28, 225-227) identify many of the core features of bullying, including the common perception of the bullied individual that any action taken against the perpetrator will have a negative outcome. However, some forms of bullying are both less overt and more insidious.

Bullying in the form of career advice given either informally or formally during supervision, or the record of in-training assessment process, is harder to define but potentially just as damaging. It may affect performance, confidence and career progression. The authors identify supervision as supportive and indeed it can be, but it may also be one of the arenas of bullying. Senior clinicians naturally develop areas of expertise and bias toward certain activities, but awareness is needed of when advice moves beyond the appropriate (and perhaps directive) into an abuse of power, position or knowledge. For example, is it advice or bullying to suggest dropping union involvement from a curriculum vitae or to suggest dropping union activities altogether to secure senior posts in a chosen specialty? Essentially, the difference relates to whether the advice is sought and the consequences of not taking the advice

While it is important to eliminate discrimination, harassment and bullying, it is also essential to differentiate bullying from legitimate and reasonable management of staff performance, and indeed from robust academic debate. However, given the negative effects of bullying is it now timely for the College to take an active role in eliminating it from the profession?

\section{Declaration of interest}

Dr Alastair Hull was on the Executive of the Junior Members Forum, of the British Medical Association which organised a symposium on 'Bullying, harassment and discrimination: still rife in the 21st Century NHS' in 2003.

Alastair Hull Locum Consultant Psychiatrist Murray Royal Hospital, Muirhall Road, Perth PH2 7BH

\section{The objective structured clinical examination}

I read with interest the recent letter regarding the Part I MRCPsych OSCE (Yak et al, Psychiatric Bulletin, July 2004, 28

265-266). However, I disagree with some conclusions.

From personal experience, most candidates feel that the OSCE does provide a fairer assessment of their skills, and I do not believe that the process of dissecting skills into an OSCE format is inherently harmful to the training of future psychiatrists. There are many important clinical skills that can be comfortably demonstrated within seven minutes; first-rank symptoms must be elicited before their context can be understood.

However, I would agree that too often, time itself becomes the major hurdle. This is quite contrary to clinical practice. If a difficult patient takes longer than expected in clinic, we would not rush them out, or end prematurely, but would take the necessary time and if required the clinic would overrun. The emphasis of the exam should not be different.

I am also concerned with the progression of the type of vignettes seen in the three OSCE exams so far. From the initial, very reasonable subjects, the cases are rapidly evolving into unreasonable scenarios. How many of us saw cases of temporal lobe epilepsy during our first year in psychiatry?

The OSCE exam, therefore, is less than perfect, but at least fair. I remain more concerned about the Part II examination, where candidates struggle against the hopelessness of the uncontrollable variables of patient and examiners. Perhaps it is the candidates, rather than the chief examiner, who adopt the mantle of Sisyphus (Tyrer \& Oyebode, British Journal of Psychiatry, March 2004, 184, 197-199).

Owen Haeney Senior House Officer to Professor Oyebode, Queen Elizabeth Psychiatric Hospital, Mindelsohn Way, Edgbaston, Birmingham B15 2QZ

\section{The use of 'drug dogs' in psychiatry}

This 'opinion and debate' (Gordon \& Haider, Psychiatric Bulletin, June 2004, 28, 196-198) appeared in the Bulletin within weeks of our local site teaching a seminar on 'Safer Services'. Concurrently, our local paper's front page feature was entitled 'Sniffer dogs for St Luke's wards', and contained a photograph of and many quotes from our Chief Executive. I took the opportunity to circulate a brief questionnaire to attenders of our multiprofessional site teaching. Nine questionnaires were returned, eight from medical staff and one from nursing staff.

Views about random visits from sniffer dogs and their handlers included those that it would be a waste of money, would create an atmosphere of fear and distrust, would be counter-therapeutic, and may be an embarrassment to those patients (and staff) identified by sniffer dogs. However, they would reduce illicit substance misuse and dealer activities, could prevent non-users being introduced to drugs, may have an educational effect by promoting 'zero-tolerance', and knowing who is using illicit drugs could inform prescribing for those patients. There were concerns about consequences such as implementing prosecutions and discharge of patients/dealers that may not be therapeutic. There was also concern that these measures may not actually work.

Views about airport-style metal detectors at unit receptions were also solicited. Responders thought that these may reduce or prevent weapons being brought into our units, but they would require constant manning by staff with search skills (females for females), would be very expensive to maintain, would be slow for large numbers of people entering the unit at one time, e.g. students, and inconvenient for people going in and out of the unit frequently, e.g. doctors. There were also concerns about having to empty pockets, and what would happen when weapons were found - disarming people may cause violence in itself.

'Would you feel safer at work with sniffer dogs and/or metal detectors in use?' The replies were five Yes, three No, one not answered; three commented that adequate staffing levels and presence of security guards would help to make services safer for patients, visitors, and staff, and one person suggested DNA and fingerprints of all patients should be taken!

Gianetta Rands Consultant Psychiatrist and College Tutor, Highgate Mental Health Centre, Camden and Islington Mental Health and Social Care Trust, London N195JG, and Honorary Senior Lecturer, Department of Mental Health Sciences, Royal Free and UCL Medical School.

E-mail: gianetta.rands@candi.nhs.uk 